\title{
Bayesian inference for orbital eccentricities
}

\author{
L. B. Lucy
}

\author{
Astrophysics Group, Blackett Laboratory, Imperial College London, Prince Consort Road, London SW7 2AZ, UK \\ e-mail: 1.lucy@imperial.ac.uk
}

Received 14 June 2012 / Accepted 21 December 2012

\section{ABSTRACT}

\begin{abstract}
Highest posterior density intervals (HPDIs) are derived for the true eccentricities $\varepsilon$ of spectroscopic binaries with measured values $e \approx 0$. These yield upper limits when $e$ is below the detection threshold $e_{\text {th }}$ and seamlessly transform to upper and lower bounds when $e>e_{\mathrm{th}}$. In the main text, HPDIs are computed with an informative eccentricity prior representing orbital decay due to tidal dissipation. In an appendix, the corresponding HPDIs are computed with a uniform prior and are the basis for a revised version of the Lucy-Sweeney test, with the previous outcome $\varepsilon=0$ now replaced by an upper limit $\varepsilon_{\mathrm{U}}$. Sampling experiments with known prior confirm the validity of the HPDIs.
\end{abstract}

Key words. binaries: spectroscopic - methods: statistical - methods: data analysis

\section{Introduction}

For over two centuries, astronomers have been able to detect and analyse orbital motions for objects beyond the solar system. From measured positions (visual binaries) or radial velocities (spectroscopic binaries, exoplanets), orbital elements and their standard errors are typically obtained by least-squares. Accordingly, at this late date, when orbital motion is detected, prior information concerning similar objects is available and can be incorporated into the analysis.

An example is the Lucy-Sweeney (1971; LS) test for the statistical significance of small measured eccentricities $e$ for spectroscopic binaries (SB's). The prior information that provided support for the LS test was as follows:

1) The small $e$ 's (typically $\lesssim 0.05$ ) of numerous catalogued SB's were $\sim E(e \mid 0)$, the expected value of $e$ due to measurement errors when the true value is $\varepsilon=0$.

2) Savedoff's (1951) investigation of $e \cos \omega$, where $\omega$ is the longitude of periastron. If an SB is also an eclipsing binary (EB), $e \cos \omega$ can be determined from the velocity curve $(s p)$ and independently from the light curve (ph). For EB's with secondary eclipses midway between consecutive primary eclipses, $(e \cos \omega)_{\mathrm{ph}}=0$, but the values of $(e \cos \omega)_{\mathrm{sp}}$ are scattered over the interval $(-0.05,0.05)$, confirming that non-zero $e$ 's $\$ 0.05$ are often spurious.

3) Tidal dissipation gives $\varepsilon \rightarrow 0$ as $t \rightarrow \infty$ since, for two point masses, $\varepsilon=0$ is the state of minimum orbital energy for fixed orbital angular momentum. Thus, if the time constant for this decay is short enough, the system is likely to be observed when $\varepsilon \ll E(e \mid 0)$ - i.e., well below the measurement threshold.

In view of this prior information, $\mathrm{LS}$ adopted $\varepsilon=0$ as the preferred (null) hypothesis $\left(H_{0}\right)$ and imposed a moderately demanding level of significance before rejecting $H_{0}$ and accepting an elliptical orbit.

Given that secular evolution due to tidal dissipation was already well established in 1971 and is not less so now, there is merit in explicitly incorporating this mechanism into the analysis rather than implicitly via the LS preference for $\varepsilon=0$. This can be achieved by replacing the frequentist approach of LS by one based on Bayes' theorem.

\section{Estimating the true eccentricity $\varepsilon$}

We suppose that radial velocities of a single-lined spectoscopic binary (SB1) have been analysed to estimate the orbital elements and their standard errors. We ask: what can be inferred about the error-free elements when orbital evolution due to tidal dissipation is taken into account?

\subsection{Posterior probability}

We adopt the notation used in Lucy (1974; L74). The vectors of the estimated and the error-free elements are denoted by $\boldsymbol{x}$ and $\boldsymbol{\xi}$, respectively; and the distribution of probability in $x$-space for given $\boldsymbol{\xi}$ is denoted by $\Pi(\boldsymbol{x} \mid \boldsymbol{\xi}) \mathrm{d} \boldsymbol{x}$. Integrating over $\boldsymbol{\xi}$-space, we find that the distribution of probability in $\boldsymbol{x}$-space is $\phi(\boldsymbol{x}) \mathrm{d} \boldsymbol{x}$, where

$\phi(\boldsymbol{x})=\int \psi(\boldsymbol{\xi}) \Pi(\boldsymbol{x} \mid \boldsymbol{\xi}) \mathrm{d} \boldsymbol{\xi}$

Here $\psi$ is the probability density function (pdf) that represents our prior knowledge about $\psi(\xi) \mathrm{d} \xi$, the distribution of probability in $\xi$-space for the SB1's true elements.

Since SB1's with small $e$ 's are of interest, we suppose that Sterne's (1941) elements have been chosen, as in LS. The six elements are then: $P$, the orbital period; $\gamma$, the systemic velocity; $K$, the semi-amplitude of the velocity curve; $T_{0}$, an epoch at which the mean longitude is zero; and the pair $e \cos \omega, e \sin \omega$. In terms of these parameters, the radial velocity curve of an SB1 is given by Eq. (1) in LS.

With this choice and the assumption $e^{2} \ll 1$, the off-diagonal elements of the least-squares matrix have zero expectation values when observational weight is uniformly distributed in phase (LS). Since observers strive to meet this condition, we assume 
it to be true; and this then implies negligible correlations between the elements. Accordingly, to a good approximation, the error-broadening kernel $\Pi(\boldsymbol{x} \mid \boldsymbol{\xi})$ is simply the product of the six Gaussians giving the independent error distributions of the six elements.

Next consider the pdf $\psi(\xi)$, which is convolved in Eq. (1) with the kernel $\Pi(\boldsymbol{x} \mid \boldsymbol{\xi})$. It follows that $\psi$ is only relevant if it varies significantly within the error bars of an individual orbital element. This is not true for $P, \gamma, K$, and $T_{0}$. However, when orbit circularisation is taken into account, $\psi(\boldsymbol{\xi})$ may vary significantly within the domain $\left(e \cos \omega \pm \sigma_{e \cos \omega}, e \sin \omega \pm \sigma_{e \sin \omega}\right)$. Accordingly, we now integrate Eq. (1) with respect to the $\left(P, \gamma, K, T_{0}\right)$-components of $\boldsymbol{x}$ and use the normalization of $\Pi(\boldsymbol{x} \mid \boldsymbol{\xi}) \mathrm{d} \boldsymbol{x}$ with respect to each of these four elements. The result is

$\phi_{\mathrm{c}}(x, y)=\int \psi_{\mathrm{c}}(\xi, \eta) \Pi_{\mathrm{c}}(x-\xi, y-\eta) \mathrm{d} \xi \mathrm{d} \eta$

where $x=e \cos \omega$ and $y=e \sin \omega$ are the two surviving estimated quantities, whose error-free values are $\xi=\varepsilon \cos \varpi$ and $\eta=\varepsilon \sin \varpi$, respectively, and the subscript $c$ indicates that the pdf's are defined on a Cartesian grid.

We now assume that the standard errors of $e \cos \omega$ and $e \sin \omega$ are equal, which is true of their expectation values when observational weight is uniformly distributed in phase (LS). The broadening kernel is then the circular normal distribution

$\Pi_{\mathrm{c}}(x-\xi, y-\eta)=\frac{1}{2 \pi \mu^{2}} \exp \left[-\frac{(x-\xi)^{2}+(y-\eta)^{2}}{2 \mu^{2}}\right]$

where $\mu=\sigma_{e \cos \omega}=\sigma_{e \sin \omega}$.

Bayes' theorem can now be invoked to derive - see Eq. (10) in $\mathrm{L} 74$ - the posterior probability

$Q_{\mathrm{c}}(\xi, \eta \mid x, y)=\frac{\psi_{\mathrm{c}}(\xi, \eta) \Pi_{\mathrm{c}}(x-\xi, y-\eta)}{\phi_{\mathrm{c}}(x, y)}$.

Thus, if the least-square solution is

$x=e \cos \omega \pm \mu, \quad y=e \sin \omega \pm \mu$

the distribution of probability in $(\varepsilon \cos \varpi, \varepsilon \sin \varpi)$-space is given by $Q_{\mathrm{c}}(\xi, \eta \mid x, y) \mathrm{d} \xi \mathrm{d} \eta$. To evaluate this posterior distribution, we must specify what our expectations were for $\psi_{\mathrm{c}}(\xi, \eta) \mathrm{d} \xi \mathrm{d} \eta$, the prior distribution of probability in $(\varepsilon \cos \varpi, \varepsilon \sin \varpi)$-space.

For the problem at hand, polar $(p)$ coordinates $(\varepsilon, \varpi)$ are more convenient than the Cartesian coordinates $(\xi, \eta)$ in the above formulae. The Jacobian of the transformation is $J=\varepsilon$, so that, by conservation of probability,

$\psi_{\mathrm{p}}(\varepsilon, \varpi)=\varepsilon \psi_{\mathrm{c}}(\xi, \eta)$

and

$Q_{\mathrm{p}}(\varepsilon, \varpi \mid x, y)=\varepsilon Q_{\mathrm{c}}(\xi, \eta \mid x, y)$.

\subsection{A physical model for $\psi_{\mathrm{p}}$}

Given $P$, there is a $2 \mathrm{D}$ family of binaries that match the measured $K$ at some inclination. For simplicity, a representative example is chosen to avoid integrating over all possibilities.

Now consider an ensemble of such binaries that form at a uniform rate in the solar neighbourhood and have lifetime $t_{*}$. We further suppose that all have $\varepsilon=\varepsilon_{0}$ at $t=0$ and that thereafter $\varepsilon$ decays exponentially with fixed $e$-folding time $t_{*} / v$, so that

$\ln \varepsilon(t)=\ln \varepsilon_{0}-v\left(\frac{t}{t_{*}}\right)$.
It follows that $\ln \varepsilon$ is uniformly distributed in the interval $\left(\ln \varepsilon_{*}, \ln \varepsilon_{0}\right)$, where $\varepsilon_{*}=\varepsilon_{0} \exp (-v)$. The probability that $\varepsilon \in$ $(\varepsilon, \varepsilon+\mathrm{d} \varepsilon)$ is therefore $\mathrm{d} \varepsilon / v \varepsilon$.

If we now assume randomly oriented orbits, the probability that $\varpi \in(\varpi, \varpi+\mathrm{d} \varpi)$ is $\mathrm{d} \varpi / 2 \pi$. Accordingly, the prior probability that a binary is in the element d $\varepsilon d \varpi$ at $(\varepsilon, \varpi)$ is $\psi_{\mathrm{p}} \mathrm{d} \varepsilon \mathrm{d} \varpi$, where

$\psi_{\mathrm{p}}(\varepsilon, \varpi)=\frac{1}{2 \pi v \varepsilon}$ for $\varepsilon \in\left(\varepsilon_{*}, \varepsilon_{0}\right)$.

Note that $\psi_{\mathrm{p}}$ decreases with increasing $v=\ln \left(\varepsilon_{0} / \varepsilon_{*}\right)$. Nevertheless, normalization of this pdf is maintained by the corresponding decrease in $\varepsilon_{*}$, the lower limit for integrations over $\varepsilon$.

In introducing this physical model, we in effect adopt an informative prior. The following quote is apt: "The real power of Bayesian inference lies in its ability to incorporate "informative" prior information, not "ignorance"” (Feldman \& Cousins 1998).

\subsection{Distribition of $\varepsilon$}

For numerical calculations, it is convenient to transform the integral in Eq. (2) into an integration with respect to the polar coordinates $\varepsilon$ and $\varpi$, so that

$\phi_{\mathrm{c}}(x, y)=\int_{\varepsilon_{*}}^{\varepsilon_{0}} \int_{0}^{2 \pi} \psi_{\mathrm{p}}(\varepsilon, \varpi) \Pi_{\mathrm{c}}(x-\xi, y-\eta) \mathrm{d} \varepsilon \mathrm{d} \varpi$

and to use logarithmic spacing in $\varepsilon$ in order to accurately evaluate the contribution near $\varepsilon_{*}$. Note that $\phi_{\mathrm{c}}$ is independent of $\omega$ when $\psi_{\mathrm{p}}$ is independent of $\varpi$, as in Eq. (9).

With $\phi_{\mathrm{c}}$ evaluated, the distribution of probability in $(\varepsilon, \varpi)$ space is given by $Q_{\mathrm{p}}(\varepsilon, \varpi \mid x, y) \mathrm{d} \varepsilon \mathrm{d} \varpi$, with $Q_{\mathrm{p}}=\varepsilon Q_{\mathrm{c}}$ from Eq. (4). This 2D pdf, which in general is not independent of $\varpi$, may be of interest when analysing a particular SB1. But here our interest is in $\varepsilon$, so we integrate over $\varpi$ to obtain

$q(\varepsilon \mid e)=\int_{0}^{2 \pi} Q_{\mathrm{p}}(\varepsilon, \varpi \mid x, y) \mathrm{d} \varpi$.

Note that $q(\varepsilon \mid e)$ is independent of $\omega$ because of the absence of a correlation term in $\Pi_{\mathrm{c}}-$ see Eq. (3). This in turn follows from the assumptions (Sect. 2.1) that $e^{2} \ll 1$ and that observational weight is uniformly distributed in phase.

The posterior probability that the true eccentricity $\in(\varepsilon, \varepsilon+$ $\mathrm{d} \varepsilon$ ) is therefore $q(\varepsilon \mid e) \mathrm{d} \varepsilon$, with mean value

$\langle\varepsilon\rangle=\int_{\varepsilon_{*}}^{\varepsilon_{0}} \varepsilon q(\varepsilon \mid e) \mathrm{d} \varepsilon$.

\subsection{Bayesian terminology}

In the above, notation and terminology is from L74. To modern Bayesians, $\Pi_{\mathrm{c}}(x-\xi, y-\eta)$ is the likelihood and $\phi_{\mathrm{c}}(x, y)$ is the Bayes' factor. Elsewhere, modern usage is followed with respect to the terms prior pdf, posterior pdf and credible intervals.

\section{Numerical results}

The theory of Sect. 2 is now illustrated by computing a particular case in detail. 


\subsection{Parameters}

There are two basic parameters, $e / \mu$ and $\nu$, the number of e-folding decay times in $t_{*}$.

Note that $\epsilon_{0}$ is not a consequential parameter provided that $e \ll \epsilon_{0}$. In effect, we assume that an SB1 with $e \approx 0$ has reached this configuration due to secular evolution and not due to the formation mechanism. In these calculations, $\epsilon_{0}=0.5$.

We choose $v=8.52$, so that $\varepsilon_{*}=10^{-4}$. Then, with $\mu=0.01$, $\varepsilon(t)<2.45 \mu$, the LS threshold, when $t>0.35 t_{*}$. Thus, from our ensemble of SB1's, $\approx 65 \%$ would be assigned $\varepsilon=0$ by the LS test.

\subsection{The posterior $p d f \chi(\log \varepsilon \mid e)$}

Because of the concentration of probability towards $\varepsilon_{*}$, plots are more informative if the abscissa is $\log \varepsilon$ rather than $\varepsilon$. Accordingly, we define

$\chi(\log \varepsilon \mid e)=\varepsilon q(\varepsilon \mid e) \times \ln 10$.

In Fig. 1, this pdf is plotted for $e / \mu=1.0,2.45,3.03$ and 3.72, values selected as follows: if $\varepsilon=0$, LS showed that the probability $p_{\mathrm{e}}$ of exceeding $e$ is given by

$\ln p_{\mathrm{e}}=-\frac{1}{2}\left(\frac{e}{\mu}\right)^{2}$

provided that $\mu \ll 1$. Therefore, when testing $H_{0}$, the above values of $e / \mu$ correspond to levels of significance $61,5,1$ and $0.1 \%$, respectively. The criterion $e / \mu>1$ was proposed and implemented by Luyten (1936); the 5\% level by LS.

For $e / \mu=3.72, \chi$ is an asymmetric bell-shaped function peaking at $\approx e$, but with a tail extending down to $\log \varepsilon_{*}=-4.0$. As $e / \mu$ decreases, the peak weakens and the tail strengthens. At $e / \mu=1.0$, the peak is absent and all the probability is in the tail, which derives from the physical model. Intermediate calculations show that the peak first appears at $e / \mu=1.42$. Thus, for $e / \mu<1.42, \chi$ is a monotonically decreasing function of $\varepsilon$. For $e / \mu>1.42, \chi$ is unimodal.

The pdf $\chi$ for $e / \mu=3.72$ and $\mu=0.01$ in Fig. 1 is computed with $\varepsilon_{0} / \mu=50$. Repeating this calculation shows that $\chi$ is independent of the upper limit $\varepsilon_{0}$ provided that $\varepsilon_{0} / \mu \gtrsim 10$.

\subsection{Percentiles}

The posterior pdf's in Fig. 1 imply asymmetric and rapidly changing credible (or Bayesian confidence) intervals as $e / \mu$ varies. These are plotted in Fig. 2 for the indicated values of the probability that the true eccentricity is $<\varepsilon$. For the normal distribution, the values $0.159,0.500,0.841$ and 0.977 correspond to displacements of $-1,0,+1$ and $+2 \sigma$, respectively.

Figure 2 reveals a dramatic switch in solution regime at $e_{\dagger} / \mu \approx 3.6$. For $e \gtrsim e_{\dagger}$, the measured value $e$ is close to the $50 \%$ percentile and is tightly enclosed by the $\pm 1 \sigma$ intervals. In this regime, inferences are dominated by the actual measurement $e \pm \mu$. But this ceases to be so for $e \lesssim e_{\dagger}$. Thus, for $e / \mu<2.65$, the "solution" $\varepsilon=e$ falls outside the $\pm 1 \sigma$ intervals. Evidently, for $e \lesssim e_{\dagger}$, inferences are increasingly dominated by the model of Sect. 2.2.

\subsection{Highest posterior density intervals}

For $e / \mu<1.42, \chi$ is a monotonically decreasing function of $\varepsilon$ (Sect. 3.2). It follows that traditional, equal-tail credible

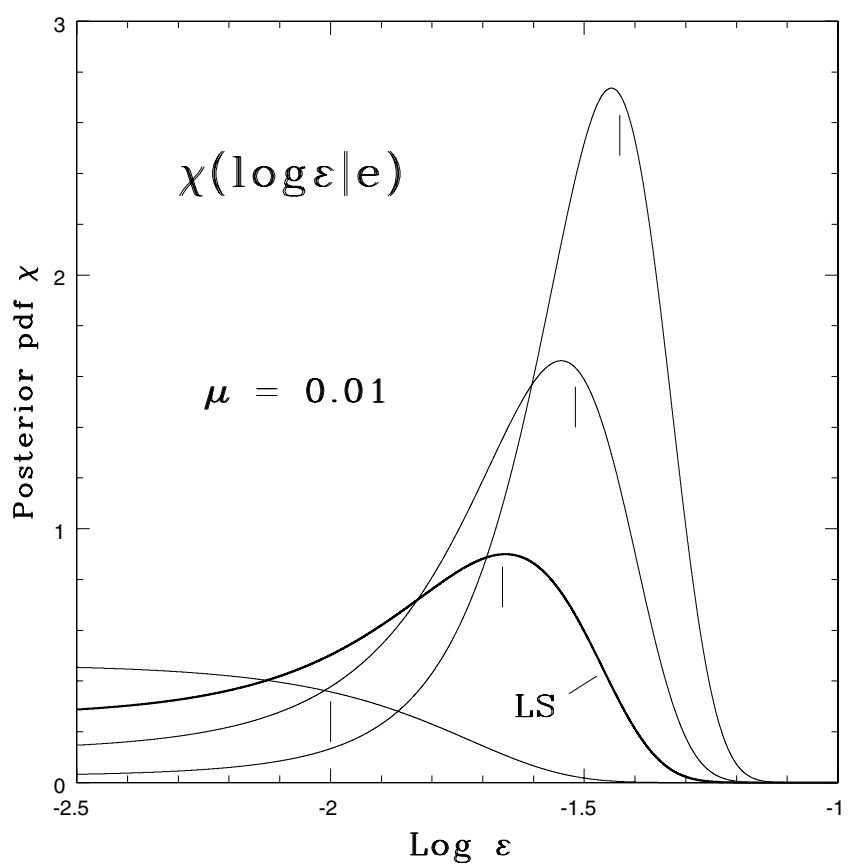

Fig. 1. The pdf $\chi(\log \varepsilon \mid e)$ for the values of $e$ indicated by the vertical lines. These are at $e / \mu=1.00,2.45,3.03$ and 3.72, corresponding to levels of significance of $61,5,1$, and $0.1 \%$, respectively. The bold curve is the pdf at the point where the LS-test switches from accepting to rejecting a circular orbit.

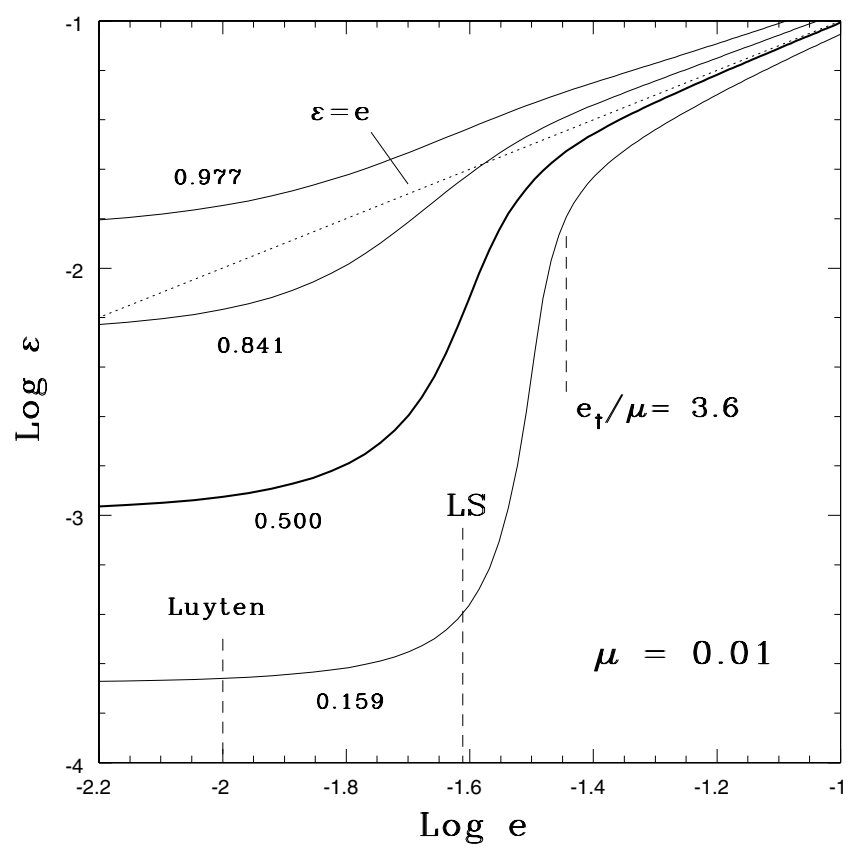

Fig. 2. Credible intervals for the posterior pdf $\chi(\log \varepsilon \mid e)$ as functions of $e$. The plotted boundaries correspond to the true eccentricity being $<\varepsilon$ with the indicated probabilities. The values of $e$ corresponding to levels of significance $61 \%$ (Luyten) and 5\% (LS) are indicated, as is the regime transition at $e_{\dagger}$.

intervals exclude the point $\left(\varepsilon=\varepsilon_{*}\right)$ with greatest probability density (pd). This undesirable feature is avoided by instead computing highest posterior density intervals (HPDI; Box \& Tiao 1973). These intervals are such that every point included has a higher pd than every point excluded. 


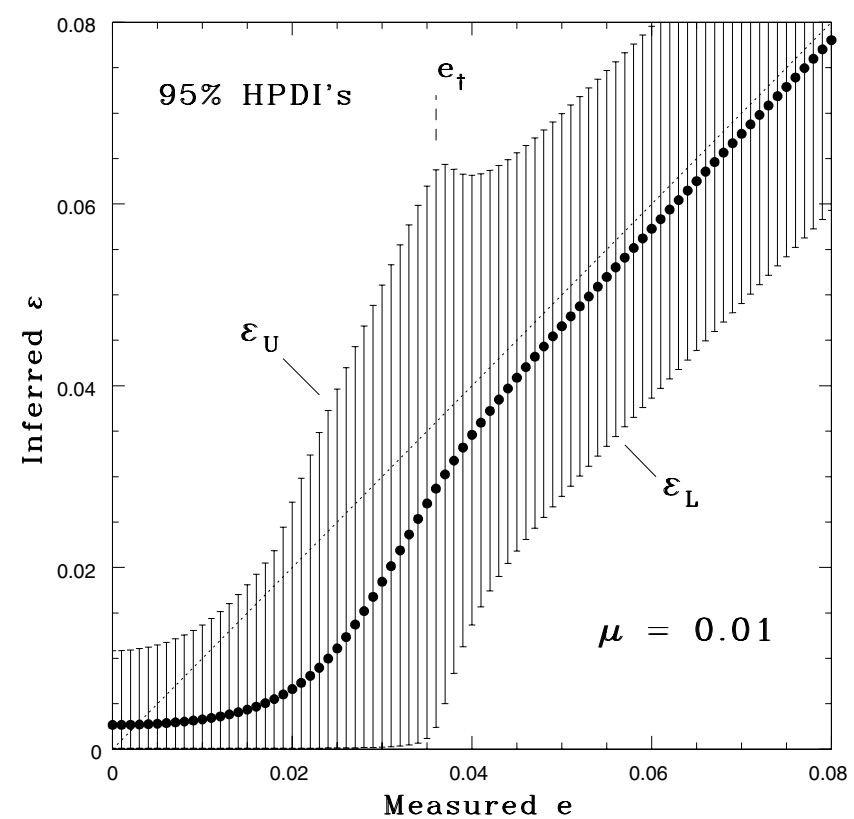

Fig. 3. Highest posterior density intervals for the posterior pdf $\chi(\log \varepsilon \mid e)$. The enclosed posterior probability of each HPDI is $95 \%$, and the prior pdf $\psi$ is given by Eq. (9). The filled circles are the posterior means $\langle\varepsilon\rangle$ computed from Eq. (12).

In general, the calculation of HPDIs is non-trivial. But here the pdf's $\chi$ are not pathological (Fig. 1), and so the following clipping algorithm finds the HPDI for specified $e$ and designated enclosed probability $(1-\alpha)$.

Let $\log \varepsilon_{k_{\mathrm{L}}}, \ldots, \log \varepsilon_{k_{\mathrm{U}}}$ be consecutive grid points that belong to and define the HPDI $\left(\varepsilon_{k_{\mathrm{L}}}, \varepsilon_{k_{\mathrm{U}}}\right)$. Then an HPDI with smaller included probability is obtained by eliminating the grid point $\log \varepsilon_{k_{\mathrm{L}}}$ if $\chi_{k_{\mathrm{L}}}<\chi_{k_{\mathrm{U}}}$ or the grid point $\log \varepsilon_{k_{\mathrm{U}}}$ if $\chi_{k_{\mathrm{U}}}<\chi_{k_{\mathrm{L}}}$. This is repeated until the included probability $=(1-\alpha)$.

The 95\% HPDIs thus obtained for $\chi(\log \varepsilon \mid e)$ are plotted for $e=0.000(0.001) 0.080$ in Fig. 3. For $e<0.018$, the HPDIs are effectively one-tail intervals since the lower bound is $\varepsilon_{\mathrm{L}}=\varepsilon_{*}=$ $10^{-4}$. Accordingly, for this problem, HPDIs provide a seamless transition from upper limits for non-detected to two-sided intervals for detected eccentricities (cf. Feldman \& Cousins 1998). This is an appealing aspect of HDPIs for interpreting measured $e$ 's and their uncertainties.

For $e<0.0142$, the interval excluded from an HPDI is a single-tail because of the aforementioned monotonicity. For $e>$ 0.0142 , the pdf $\chi$ is unimodal (Fig. 1), with a maximum whose location $\rightarrow e$ as $e / \mu$ increases. This emerging, measurementdriven maximum eventually brings about the transition from one- to two-tailed intervals. For $e>0.018$, the excluded probability is contained in two tails, with the upper tail's probability being initially $5 \%$, but this decreases to $\approx 2.5 \%$ when $e / \mu \gg 1$ because of $\chi$ 's increasing symmetry - see Fig. 1.

Note that for the normal distribution, $\mathcal{N}(0,1)$, the $95 \%$ HPDI is the familiar equal tail interval $(-1.96,+1.96)$ and the width of this interval is the narrowest that ecloses $95 \%$ of the probability. For non-symmetric pdf's, HPDIs are the narrowest intervals enclosing probability $(1-\alpha)$ and as such are a natural generalization of the conventional equal-tail intervals for symmetric, bell-shaped pdf's.

Confidence intervals are an economical means of conveying the compactness or otherwise of a variate's distribution. The resulting loss of information, if of concern, can be avoided by plotting the pdf's, as in Figs. 1 and A.2.

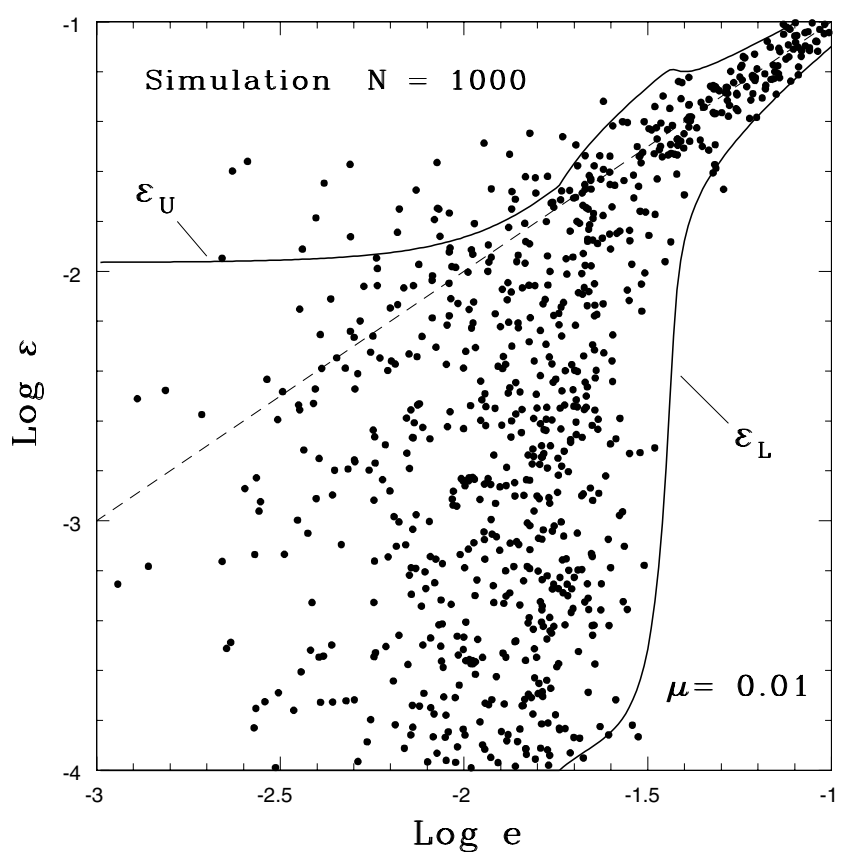

Fig. 4. Ensemble simulation comprising $N=1000$ SB1's with the parameters of Sect. 3.1. The plotted points randomly sample the time interval $\left(0, t_{*}\right)$, have true eccentricities $\varepsilon$ from Eq. (8), and have Gaussian measurement errors added to derive $e$. The $95 \%$ HPDIs $\left(\varepsilon_{\mathrm{L}}, \varepsilon_{\mathrm{U}}\right)$ are indicated. For $e<e_{\mathrm{th}}=0.018$, the lower limit $\varepsilon_{\mathrm{L}}=\varepsilon_{*}=10^{-4}$.

\subsection{Detection threshold}

The transition from one- to two-tailed HPDIs is a natural definition of the detection threshold $e_{\text {th }}$ for non-zero eccentricity - i.e., the measured value $e$ above which attribution purely to measurement errors is implausible. However, Fig. 3 shows that $\varepsilon_{\mathrm{L}}$ remains $\ll e$ for a considerable interval beyond $e_{\mathrm{th}}=0.018$, which in any case depends on $v$, a parameter likely to be only crudely estimated.

If detection is crucial for a subsequent investigation - e.g., an observing program - then a threshold closer to $e_{\dagger}$ should be adopted (Figs. 2 and 3).

\subsection{Simulation}

The role that HPDIs can play in reporting eccentricities is best illustrated by sampling experiments. Accordingly, synthetic data for the model of Sect. 2.2 are created as follows: if $z_{1}, z_{2}$ are random numbers in $(0,1)$, a random ensemble member in $(\varepsilon, \varpi)$ space is at

$\varepsilon=\varepsilon_{0} \exp \left(-v z_{1}\right) \quad \varpi=2 \pi z_{2}$.

Then, if $\zeta_{1}, \zeta_{2}$ are random Gaussian variates, this ensemble member is observed at the point

$e \cos \omega=\varepsilon \cos \varpi+\mu \zeta_{1} \quad e \sin \omega=\varepsilon \sin \varpi+\mu \zeta_{2}$.

Repeated $N$ times, the resulting $e$ 's comprise a simulated observing campaign of $N$ random ensemble members whose exact eccentricities $\varepsilon$ are known.

With $\varepsilon_{*}=10^{-4}$, a sample of $N=1000 \mathrm{SB} 1$ 's are plotted in Fig. 4. As expected, the large majority of the points fall within the $95 \%$ HPDIs $\left(\varepsilon_{\mathrm{L}}, \varepsilon_{\mathrm{U}}\right)$. With Luyten's criterion $e>\mu, 79.5 \%$ of this sample would have their elliptical orbits accepted. But Fig. 4 clearly shows that most systems with $e \in(0.01,0.03)$ have 
Table 1. HPDI 95\% upper limits $\varepsilon_{95}$ when $e / \mu=1.0$.

\begin{tabular}{ccc}
\hline \hline $\log \varepsilon_{*}$ & $v$ & $\varepsilon_{95} / \mu$ \\
\hline-3.0 & 6.21 & 1.71 \\
-4.0 & 8.52 & 1.37 \\
-5.0 & 10.82 & 1.15 \\
-6.0 & 13.12 & 0.98 \\
-7.0 & 15.42 & 0.85 \\
-8.0 & 17.73 & 0.74 \\
-9.0 & 20.03 & 0.65 \\
\hline
\end{tabular}

$\varepsilon<0.01$ and so exceed Luyten's criterion because of the bias of the non-negative estimator $e$ (LS). With the $\mathrm{LS}$ criterion $e>$ $2.45 \mu$, the accepted percentage drops to 40.5, and most of the systems with $e \in(0.01,0.03)$ would now be assigned $\varepsilon=0$, a marked improvement.

The further improvement provided by the HPDIs is that the assignment $\varepsilon=0$ can now be replaced by an upper limit. Thus, for example, with this choice of prior, an SB1 with $e=$ $0.01 \pm 0.01$ is preferably reported as $\varepsilon<\varepsilon_{95}=0.014$. From the standpoint of testing theories of tidal dissipation, an upper limit is more informative than $\varepsilon=0$.

The sampling procedure can also be used to validate the upper limits. A sample with $N=10^{6}$ has 161,521 systems with $\log e \in(-2.1,-1.9)$ and 8,317 of these have $\varepsilon>\varepsilon_{95}$. Thus $94.85 \%$ lie below the upper limit, closely agreeing with the designated $95 \%$.

\subsection{Upper limits}

Upper limits $\varepsilon_{\mathrm{U}}$ when $e<e_{\mathrm{th}}$ are the most useful products of this Bayesian machinery. With their validity confirmed above, their dependence on the orbital decay rate is now explored.

Consider the representative measurement $e=0.01 \pm 0.01$, so that $e / \mu=1.0$, well below the detection threshold. In Table 1, the HPDI $95 \%$ upper limits $\varepsilon_{95}$ are given for this $e / \mu$ as a function of $\varepsilon_{*}$. The decrease of $\varepsilon_{95}$ with increasing $v$ reflects statistical reality: if the $e$-folding time $t_{*} / v \ll t_{*}$, most systems will have $\varepsilon \ll e$ and a correspondingly small $\varepsilon_{95}$. Note that $\varepsilon_{95}$ even drops below $e$ when $v \gtrsim 13$.

In analysing an observed system without a good estimate of $v$ or, equivalently, of $\varepsilon_{*}$, the conservative approach is to suppose that $\varepsilon_{*}$ is no more than a factor $\sim 10$ below the measured $e$, thus avoiding claiming too low an upper limit $\varepsilon_{\mathrm{U}}$. An even more conservative approach is to adopt a uniform prior - see Appendix.

\section{Conclusion}

In this paper, by incorporating a model of an SB1's secular evolution, Bayes' theorem is used to infer bounds on its exact eccentricity $\varepsilon$ given its measured value $e \pm \mu$. Because the system's lifetime $t_{*}$ is finite, the asymptote $\varepsilon=0$ is never reached. Thus, in contrast to Luyten (1936) and LS, the statistical problem is not one of model selection. Systems assigned $\varepsilon=0$ by these earlier tests should preferably have upper limits $\varepsilon_{\mathrm{U}}$ computed.

As Fig. 4 shows, the Bayesian upper limits contain the systems for which $e$ is significantly larger than $\varepsilon$ due to measurement errors and bias. Thus, a major historical cause of spurious $e$ 's is eliminated. But physical causes remain, such as those due to proximity effects or to additional line absorption by gas streams. An example is $\zeta$ TrA, for which Skuljan et al. (2004) improved the precision of the radial velocities by a remarkable factor of 100 and reported a small but highly significant
Table A.1. Detection thresholds for $e$.

\begin{tabular}{lcc}
\hline \hline$\alpha(\%)$ & $e_{\mathrm{LS}} / \mu$ & $e_{\mathrm{th}} / \mu$ \\
\hline 31.7 & 1.52 & 1.71 \\
10.0 & 2.15 & 2.21 \\
$\mathbf{5 . 0}$ & $\mathbf{2 . 4 5}$ & $\mathbf{2 . 4 9}$ \\
1.0 & 3.03 & 3.06 \\
0.1 & 3.72 & 3.74 \\
\hline
\end{tabular}

$e=0.0140 \pm 0.0002$. However, the significant non-detection of the Keplerian third harmonic (Lucy 2005) invalidated this claim $^{1}$. As precision improves, similar testing for the third and higher Keplerian harmonics is essential for confirming that an orbit is truly eccentric. In addition, an update of Savedoff's (1951) work would provide numerous examples of spurious $e$ 's for investigation into physical causes other than measurement bias.

Acknowledgements. I am grateful to the referee for pointing out an error in statistical terminology.

\section{Appendix A: Uniform prior}

The model of Sect. 2.2 is not appropriate if the SB or star-planet system has additional components causing significant gravitational perturbations. In this circumstance, a sensible option is to assume a uniform prior for $\varepsilon$, as is already common practice for exoplanets (e.g., Ford 2006; Eastman et al. 2013). Together with the assumption of randomly oriented orbits, the prior probability of the system being in $\mathrm{d} \varepsilon \mathrm{d} \varpi$ is then $\psi_{\mathrm{p}} \mathrm{d} \varepsilon \mathrm{d} \varpi$, where

$\psi_{\mathrm{p}}(\varepsilon, \varpi)=\frac{1}{2 \pi}$

which now replaces Eq. (9) in Sects. 2.3 and 3.

From the resulting posterior pdf $q(\varepsilon \mid e)$, the $95 \%$ HPDIs and means $\langle\varepsilon\rangle$ are plotted in Fig. A.1 for $e \in(0.00,0.08)$. Comparison with Fig. 3 shows that the HPDIs are nearly identical for $e / \mu \gtrsim 5$. However, for $e / \mu \lesssim 2$-i.e., in the non-detection domain - the upper limits $\varepsilon_{U}$ in Fig. 3 are markedly lower, reflecting the effect of tidal circularisation in creating systems with $\varepsilon \ll e$.

In contrast with Fig. 3, Fig. A.1 shows that a uniform prior results in a sharply defined detection threshold $e_{\mathrm{th}} / \mu=2.49$, which is gratifyingly close to the (frequentist) LS value $e_{\mathrm{LS}} / \mu=$ 2.45 given by Eq. (14) for $p_{\mathrm{e}}=0.05$. The thresholds for other critical levels are given in Table A.1, with the corresponding upper limits (bounds) $\varepsilon_{\mathrm{U}}$ in Table A.2.

For the representative measurement $e / \mu=1.0$ of Sect. 3.7, the $95 \%$ upper limit from Table A.2 is $2.41 \mu$. This exceeds the corresponding values in Table 1, confirming that the uniform prior is the more conservative option.

\section{A.1. A revised Lucy-Sweeney test}

Even without evidence of additional components, an investigator may be reluctant to base an analysis of orbital elements on uncertain estimates of tidal decay. If so, the assumption of a uniform prior for $\varepsilon$ should be attractive. Physically, this corresponds

\footnotetext{
1 Hearnshaw et al. (2012) have just reported eleven additional nondetections.
} 


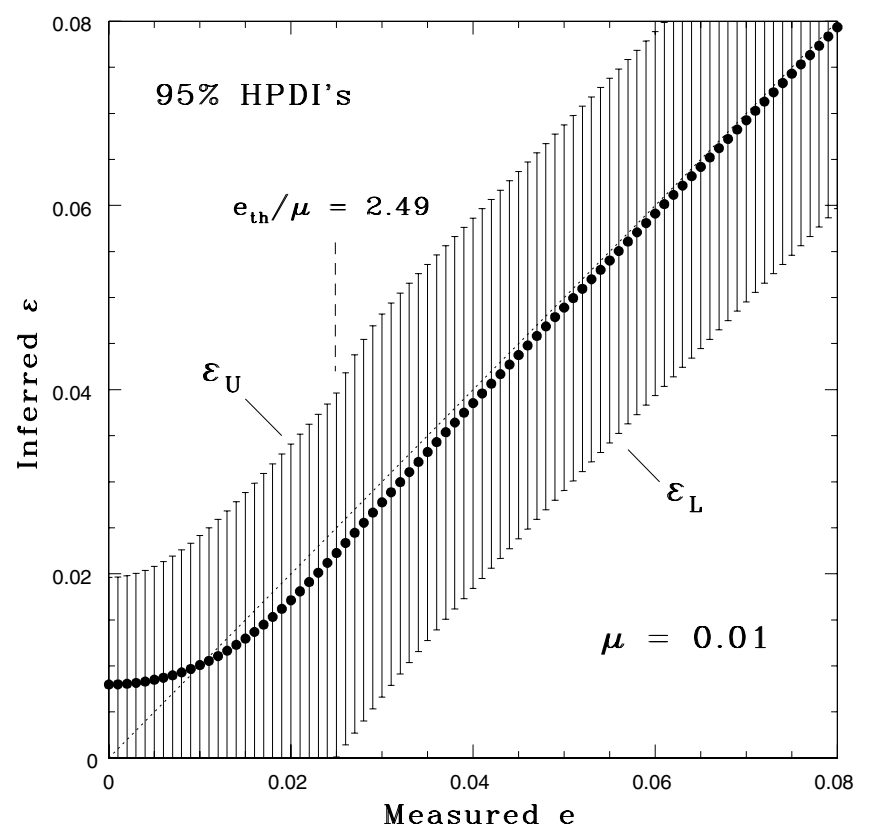

Fig. A.1. Highest posterior density intervals for the posterior pdf $q(\varepsilon \mid e)$. The enclosed posterior probability of each HPDI is $95 \%$, and the prior pdf $\psi$ is given by Eq. (A.1). The filled circles are the posterior means $\langle\varepsilon\rangle$ computed from Eq. (12).

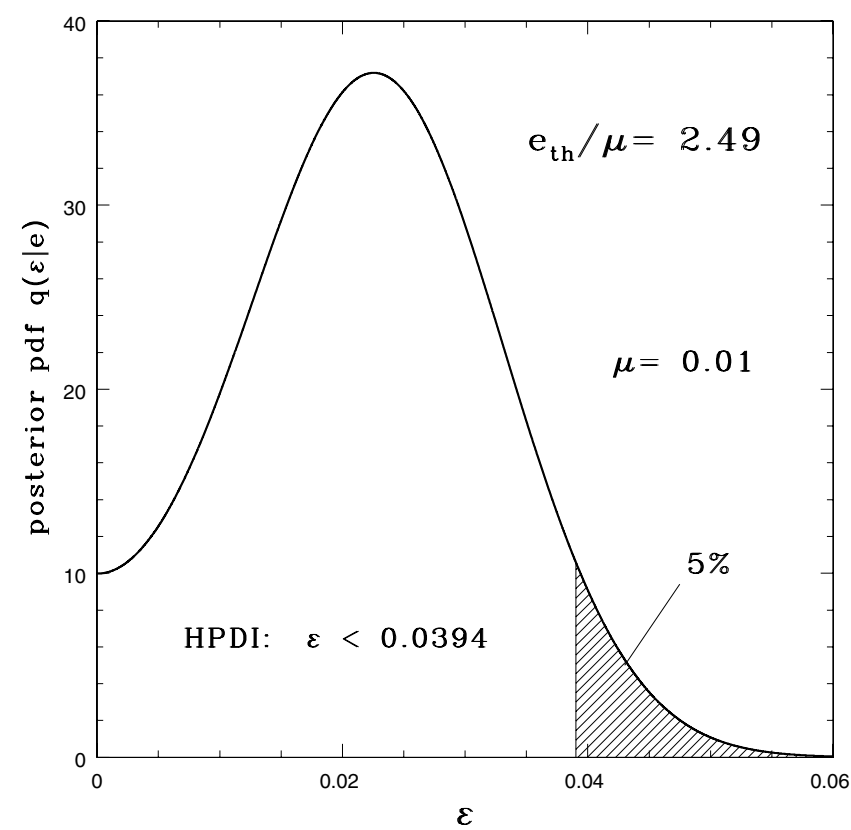

Fig. A.2. Detection threshold. The posterior pdf $q(\varepsilon \mid e)$ for $e_{\text {th }}=0.0249$, the measured value for marginal detection (Sect. 3.5). The hatched area with $\varepsilon>\varepsilon_{\mathrm{U}}=0.0394$ contains $5 \%$ of the probability. Note that $q\left(0 \mid e_{\mathrm{th}}\right)=q\left(\varepsilon_{\mathrm{U}} \mid e_{\mathrm{th}}\right)$.

to no secular evolution of $\varepsilon$ and a formation mechanism that uniformly populates the interval $0<\varepsilon<1$. Accordingly, a system with $e \approx 0$ is assumed to have formed as such (cf. Sect. 3.1).

This neutral standpoint is an attractive basis for a revised version of the LS test in which the previous acceptance of a circular orbit $\left(H_{0}\right)$ is now replaced by an upper limit.
Table A.2. Upper limits (bounds) for $\varepsilon$.

\begin{tabular}{cccccc}
\hline \hline$e / \mu$ & $\varepsilon_{68.3} / \mu$ & $\varepsilon_{90} / \mu$ & $\varepsilon_{\mathbf{9 5}} / \mu$ & $\varepsilon_{99} / \mu$ & $\varepsilon_{99.9} / \mu$ \\
\hline 0.0 & 1.00 & 1.64 & $\mathbf{1 . 9 6}$ & 2.58 & 3.29 \\
0.5 & 1.06 & 1.75 & $\mathbf{2 . 0 8}$ & 2.73 & 3.48 \\
1.0 & 1.28 & 2.05 & $\mathbf{2 . 4 1}$ & 3.11 & 3.89 \\
1.5 & 1.66 & 2.50 & $\mathbf{2 . 8 8}$ & 3.59 & 4.38 \\
2.0 & $(2.59)$ & 3.03 & $\mathbf{3 . 4 1}$ & 4.12 & 4.90 \\
2.5 & $(3.31)$ & $(3.87)$ & $\mathbf{( 3 . 9 6 )}$ & 4.65 & 5.43 \\
3.0 & $(3.86)$ & $(4.52)$ & $\mathbf{( 4 . 8 2 )}$ & 5.18 & 5.96 \\
3.5 & $(4.37)$ & $(5.04)$ & $\mathbf{( 5 . 3 6 )}$ & $(5.97)$ & 6.48 \\
4.0 & $(4.89)$ & $(5.54)$ & $\mathbf{( 5 . 8 6 )}$ & $(6.49)$ & $(7.18)$ \\
\hline
\end{tabular}

Table A.3. Upper limits $\varepsilon_{95}$ for SB1 sample.

\begin{tabular}{lccccc}
\hline \hline Star & $e$ & $\mu$ & $e / \mu$ & $\varepsilon_{95} / \mu$ & $\varepsilon$ \\
\hline YZ Cas & $(0.004)$ & 0.0037 & 1.10 & 2.50 & $<0.009$ \\
HD 7345 & $(0.046)$ & 0.019 & 2.47 & 3.93 & $<0.073$ \\
DM Per & $(0.09)$ & 0.074 & 1.22 & 2.62 & $<0.19$ \\
HD 16589 & $(0.008)$ & 0.0087 & 0.92 & 2.35 & $<0.021$ \\
HD 18337 & $(0.073)$ & 0.030 & 2.48 & 3.93 & $<0.12$ \\
HD 21912 & $(0.005)$ & 0.011 & 0.45 & 2.07 & $<0.023$ \\
\hline
\end{tabular}

The revised LS test with $1-\alpha=0.95$ proceeds as follows:

1) The eccentricity $e \pm \mu$ is derived from the least squares solution.

2) If $e / \mu>2.49$, this measured value $e \pm \mu$ is accepted.

3) However, if $e / \mu<2.49$, the measured value is replaced by the upper limit $\varepsilon_{95}$ obtained by interpolation in Col. 4 of Table A.2.

To illustrate this revised test, upper limits are given in Table A.3 for six SB1's for which circular orbits are reported on the first page of Table 1 in LS. Thus for YZ Cas, the least squares value $e=0.004$ was rejected by the LS test and $\varepsilon=0$ accepted. We now compute the $95 \%$ upper limit as follows: the estimate $\mu=0.0037$ is derived from Eq. (13) and Table 1 of LS. Linear interpolation in Table A.2 at $e / \mu=1.1$ then gives $\varepsilon_{95} / \mu=2.50$, whence $\varepsilon<\varepsilon_{95}=0.009$.

Table A.3. shows that upper limits can differ by large factors, reinforcing the earlier remark (Sect. 3.6) that upper limits are to be preferred in testing theories of tidal dissipation. A critical data base of detections and upper limits would facilitate progress in this field.

\section{References}

Box, G. E. P., \& Tiao, G. C. 1973, Bayesian Inference in Statistical Analysis, (Reading MA: Addison-Wesley)

Eastman, J., Gaudi, B. S., \& Agol, E. 2013, PASP, 125, 83

Feldman, G. J., \& Cousins, R. D. 1998, Phys. Rev. D, 57, 3873

Ford, E. B. 2006, ApJ, 642, 505

Hearnshaw, J. B., Komonjinda, S., Skuljan, J., \& Kilmartin, P. M. 2012, MNRAS, 427, 298

Lucy, L. B. 1974, AJ, 79, 745 (L74)

Lucy, L. B. 2005, A\&A, 439, 663

Lucy, L. B., \& Sweeney, M. A. 1971, AJ, 76, 544 (LS)

Luyten, W. J. 1936, ApJ, 84, 85

Savedoff, M. P. 1951, AJ, 56, 1

Skuljan, J., Ramm, D. J., \& Hearnshaw, J. B. 2004, MNRAS, 352, 975

Sterne, T. E. 1941, Proc. Natl. Acad. Sci. U.S., 27, 175 